\section{Nucleosome-binding affinity as a primary determinant of the nuclear mobility of the pioneer transcription factor FoxA}

\author{
Takashi Sekiya, ${ }_{1}^{1}$ Uma M. Muthurajan, ${ }^{2}$ Karolin Luger, ${ }^{2}$ \\ Alexei V. Tulin, ${ }^{1}$ and Kenneth S. Zaret ${ }^{1,3}$ \\ ${ }^{1}$ Epigenetics and Progenitor Cells Program, Fox Chase Cancer \\ Center, Philadelphia, Pennsylvania 19111, USA; ${ }^{2}$ Colorado State \\ University, Fort Collins, Colorado 80523, USA
}

FoxA proteins are pioneer transcription factors, among the first to bind chromatin domains in development and enable gene activity. The Fox DNA-binding domain structurally resembles linker histone and binds nucleosomes stably. Using fluorescence recovery after photobleaching, we found that FoxA1 and FoxA2 move much more slowly in nuclei than other transcription factor types, including c-Myc, GATA-4, NF-1, and HMGB1. We find that slower nuclear mobility correlates with high nonspecific nucleosome binding, and point mutations that disrupt nonspecific binding markedly increase nuclear mobility. FoxA's distinct nuclear mobility is consistent with its pioneer activity in chromatin.

Supplemental material is available at http://www.genesdev.org.

Received December 23, 2008; revised version accepted

February 18, 2009.

Transcription factors (TFs) have different activities in native chromatin. Pioneer factors bind chromatin domains early in development and help establish competence for expression states (Zaret 2002); other factors are involved in subsequent steps of gene regulation. In this study, we were interested in whether these different functional properties are reflected in differences in how the factors cycle in and out of nuclear domains. Parameters that are known to affect TF mobility in the nucleus include intrinsic affinities for DNA, chromatin modification states (Guccione et al. 2006), facilitation of chromatin binding by nucleosome remodeling complexes (Rayasam et al. 2005), interactions with other factors (Nagaich et al. 2004; Buck and Lieb 2006), promoter accessibility (Karpova et al. 2008), and diffusion in the nucleoplasm. The most common means to assess TF movement in nuclear chromatin is the fluorescence recovery after photobleaching (FRAP) technique, wherein a fluorescence-tagged protein in an area of interest is irreversibly bleached by a laser pulse. The recovery of fluorescence, by movement of unbleached protein into the bleached area, is recorded by time-lapse confocal microscopy. Generally, FRAP of chromatin components, such as

[Keywords: Chromatin; nucleus; mobility; nucleosome; FoxA factors; linker histone]

${ }^{3}$ Corresponding author.

E-MAIL zaret@fccc.edu; FAX (215) 379-4305.

Article is online at http://www.genesdev.org/cgi/doi/10.1101/gad.1775509. linker histones, is slow (e.g., $t_{1 / 2}=30-60 \mathrm{sec}$ ) (Lever et al. 2000; Misteli et al. 2000; Kimura and Cook 2001), whereas that of TFs is fast (e.g., $t_{1 / 2}=a$ few seconds) (McNally et al. 2000; Becker et al. 2002; Phair et al. 2004). Different TFs have different affinities for nucleosomal DNA, and we speculated that such differences could be a major determinant of factor mobility in the nucleus.

In this context, we focused on Fox (forkhead box) TFs, which are distinguished by a "winged helix" DNA-binding domain (DBD) that resembles linker histone in structure (Clark et al. 1993; Ramakrishnan et al. 1993). Mammals contain 43 different Fox family members, each with a DBD sequence variant that defines classes FoxA-FoxQ (Katoh 2004). The FoxA class was the first to be discovered and was found to regulate genes involved in development, signal transduction, and longevity (Katoh 2004). The winged helix DBD for FoxA factors contains a helix-turn-helix (HTH) motif that makes base-specific DNA contacts as well as two flanking loops (wings) of polypeptide chain that contact the phosphodiester backbone of DNA (Clark et al. 1993). The wing contacts increase the overall affinity of the protein for DNA (Cirillo and Zaret 2007), allowing FoxA to bind tightly on one side of DNA along the long axis, as a monomer.

Three lines of evidence indicate that FoxA factors, and likely other Fox factors, serve as pioneer factors. First, a FoxA target site at the alb1 gene enhancer is occupied in endoderm cells in which alb1 is silent, and the FoxA site remains occupied during the developmental period in which the endoderm remains competent to activate the alb1 gene (Gualdi et al. 1996; Bossard and Zaret 2000). Second, FoxA1 and FoxA2 factors are highly conserved in metazoan endoderm development (Davidson and Erwin 2006) and are redundantly necessary in the endoderm for alb1 induction and liver development (Lee et al. 2005); also, chromatin binding by FoxA1 in other contexts can enable subsequent binding by the glucocorticoid, estrogen, and androgen receptors (Grange et al. 1991; Lupien et al. 2008). Third, the structure of the Fox DBD resembles that of linker histone (Ramakrishnan et al. 1993), and FoxA factors can bind stably to a nucleosome in vitro and in vivo (Cirillo et al. 1998; Chaya et al. 2001). Such binding in vitro does not require modification of the core histones (Sekiya and Zaret 2007) and can, on highly compacted nucleosome arrays, result in hypersensitivity of the underlying nucleosome (Cirillo et al. 2002). These properties distinguish FoxA from other factors such as GATA-4, which also bind chromatin in early development (Bossard and Zaret 1998) but are dependent on FoxA for stable nucleosome binding (Cirillo and Zaret 1999). Based on these issues, we asked if FoxA factors have unusual mobility properties in the nucleus, compared with other factors, and assessed whether such properties are related to the capacity of the factors to bind nucleosomal DNA. The distinctive features we found for FoxA are considered in light of its function as a pioneer factor.

\section{Results and Discussion}

FoxA moves more slowly in nuclei than various other TF types

We first compared the fluorescence recovery of five GFPtagged factors containing DBDs of different classes (Phair 
et al. 2004): c-Myc (basic helix-loop-helix), GATA-4 (zinc finger), HMGB1 (high-mobility group), NF-1 ( $4 \times$ cys $+\alpha-$ helix), and FoxA1 (winged helix). In transiently transfected H2.35 cells (Zaret et al. 1988), FoxA1 consistently exhibited the slowest FRAP, showing a half-recovery time of $\sim 14 \mathrm{sec}$, whereas the half-times for other factors were $\sim 8$ sec for c-Myc, 7 sec for GATA-4, 5 sec for NF-1, and 1 sec for HMGB1 (Fig. 1A). For all factors, fluorescence of the bleached region recovered to the levels of the unbleached region (relative fluorescence intensity $=1$ ) (Fig. 1A). While no differences in FRAP were seen between cells expressing high or low amounts of each factor (data not shown), we quantified data from cells that expressed low levels of the GFP proteins. Also, we determined the levels of protein expression by Western blotting against the common GFP epitope and normalizing for transfection efficiency (percent green cells) (Supplemental Fig.
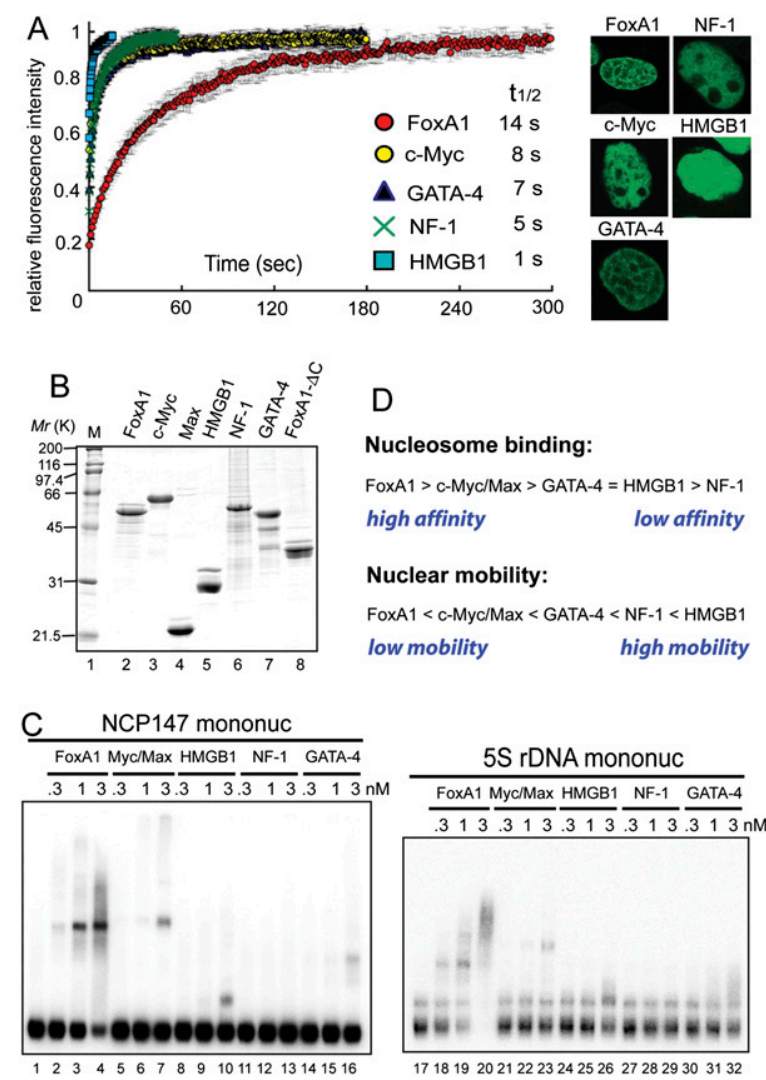

$5 S$ rDNA mononuc

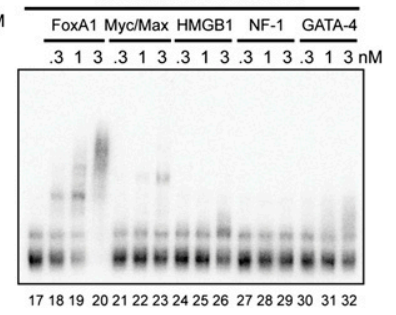

Figure 1. Low nuclear mobility correlates with high nonspecific nucleosome binding. $(A$, left $)$ FRAP of indicated GFP fusion proteins. FoxAl recovery was the slowest among the five TFs. Values represent means from at least 10 cells; standard deviations shown. The first time points were $0.24-0.5 \mathrm{sec}$ after the final bleach pulse and set as time zero. Half-recovery times $\left(t_{1 / 2}\right)$ are shown. Note that the blue triangles depicting points for GATA-4 are partially obscured by the yellow dots for c-Myc. (Right) Nuclear localization of GFP fusion proteins used in the FRAP assay. (B) SDS-PAGE of $3 \mu \mathrm{g}$ of purified proteins used in $C$. $(C)$ EMSA with increasing concentrations of purified recombinant proteins shown in $B$ and reconstituted NCP147 and 5S rDNA mononucleosomes. FoxAl showed the strongest affinity to both mononucleosomes. All shifted complexes are factors on nucleosomes; nucleosome preps were virtually free of pure DNA and complexes of factors with free DNA migrated differently (see Supplemental Fig. S2A). (D) Summary of the results from FRAP and EMSA assays.
S1A,B). HMGB1 exhibited the highest relative expression level as well as the fastest FRAP; a fast FRAP was previously recorded for HMGB1 (Phair et al. 2004). Most importantly, of the other proteins tested here, FoxA1 had slightly higher expression (Supplemental Fig. S1A,B) yet showed the slowest FRAP (Fig. 1A), indicating that nuclear protein concentration of these factors was not a primary determinant of FRAP. Furthermore, GFPFoxA1 and nontagged FoxA1 showed similar properties with regard to trans-activation (Supplemental Fig. S1CE), indicating that the attachment of GFP does not detectably affect the function of FoxA1. We also observed similarly slow mobility of FoxA2-GFP (Supplemental Fig. S1F). In conclusion, FoxA1 and FoxA2 exhibited the slowest intrinsic mobility in nuclei among the TFs tested.

\section{Movement of TFs in nuclei inversely correlates with their consensus sequence-independent binding to nucleosomes}

Because most of the eukaryotic genome is composed of nucleosomes without consensus binding sites for a given $\mathrm{TF}$, we hypothesized that the mobility of factors in nuclei could relate to their nonspecific affinity for nucleosomes. To test this, we performed electrophoretic mobility shift assays (EMSAs) of the above factors in purified, recombinant form (Fig. 1B) with nucleosomes made with 5S rDNA and NCP147 ( $\alpha$-sat) DNA, which lack target sequences for the factors and make stable, positioned nucleosomes in vitro (Simpson et al. 1985; Luger et al. 1997). FoxA1 showed the strongest affinity for both substrates, with binding to NCP147 first detectable at 0.3-1 nM compared with $3 \mathrm{nM}$ or more for c-Myc/Max, HMGB1, NF-1, and GATA-4 (Fig. 1C). On 5S rDNA mononucleosomes, $0.3 \mathrm{nM}$ FoxA1 initiated binding comparable with $3 \mathrm{nM} \mathrm{c}-\mathrm{Myc} / \mathrm{Max}$ or more of the other factors (Fig. 1C). Strikingly, the order of the nonspecific affinity of the factors for nucleosomes corresponds well to the inverse order with which the factors move in the nucleus, as assayed by FRAP (Fig. 1D). Note that the nucleosome preps were virtually devoid of free DNA and FoxA1 complexes with nucleosomes migrated distinctly from FoxA1 complexes with free DNA (Supplemental Fig. S2A), as seen previously (Cirillo et al. 1998; Cirillo and Zaret 1999). On free DNAs, FoxA1 bound about twofold better than the other factors, followed by both c-Myc/Max and HMGB1, then by GATA-4, and then by NF-1 (Supplemental Fig. S2B). Thus, nuclear mobility differences inversely correlate best with binding of the factors nonspecifically to nucleosomes and similarly but not as closely to their relative affinities for free DNA.

\section{Quantitating FoxA1 movement across the nucleus}

To better visualize and quantitate the dynamic movement of FoxA1 in the presence of native chromatin, we used a half-area FRAP assay (Phair et al. 2004). In this experiment, we bleached an approximately half-nuclear area and carefully quantitated the FRAP across three regions of the bleached domain (Fig. 2A-C). As a control, we investigated c-Myc, the second-most-stable binding factor in our above assays and previously shown to bind particular chromatin domains (Guccione et al. 2006). After bleaching, GFP-c-Myc fluorescence equilibrated 

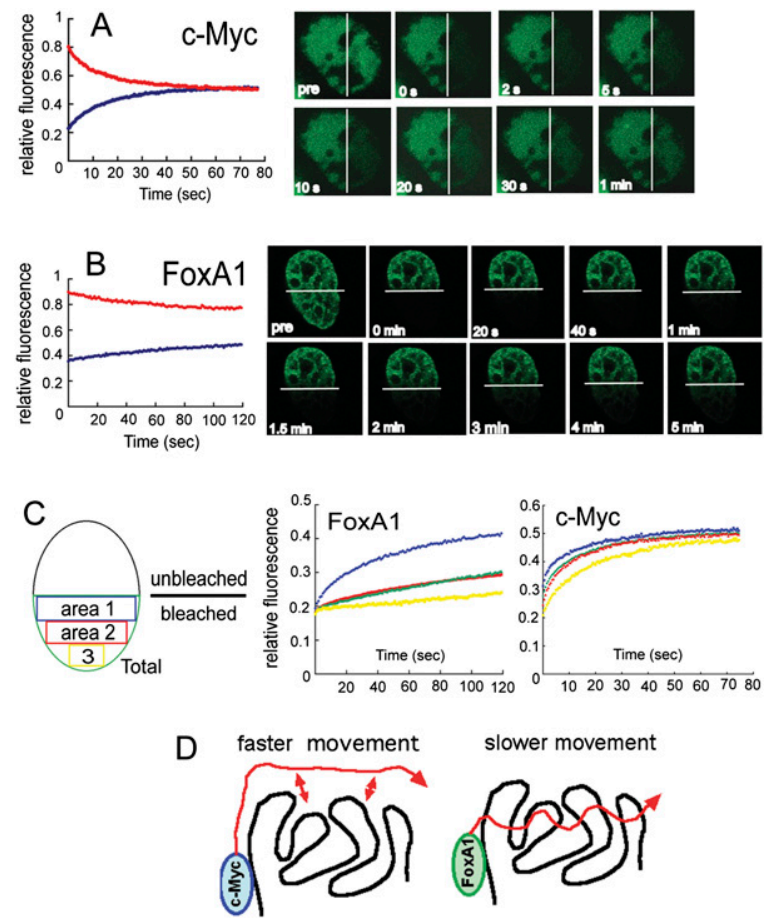

Figure 2. Markedly slow movement of FoxAl across the nucleus. (A) Half-area FRAP assay of c-Myc. (Left) Quantification of the fluorescence recoveries of c-Myc after bleaching half the nuclear area. Average fluorescence intensities in the bleached (blue circles) and unbleached (red circles) region were plotted over time. Values are averages from at least 10 cells. (Right) Fluorescent images of cMyc during the FRAP process. White bars indicate the border between the bleached and unbleached areas. Times after bleaching are indicated. $(B)$ Half-area FRAP assay of FoxAl, as in $A$. $(C)$ The average fluorescence intensities of FoxAl and c-Myc in each area were quantified and plotted. (Blue squares) Area 1; (red) area 2; (yellow) area 3; (green) total bleached region. $(D)$ Different movement models for c-Myc and FoxAl. (Left) c-Myc interaction with chromatin is less stable, with more repeated association and dissociation allowing more extensive diffusion in the nucleoplasm. (Right) The higher chromatin affinity of FoxA1 keeps it more closely associated with chromatin, causing slower overall movement.

between the bleached and unbleached areas in about $40-$ $50 \mathrm{sec}$ (Fig. 2A), as reported previously (Phair et al. 2004). Unbleached GFP-c-Myc reached the distal part of the bleached nucleus within $5 \mathrm{sec}$, and, notably, the entire bleached domain recovered nearly uniformly (Fig. 2A,C). Taken together, our results indicate that c-Myc's low nucleosome-binding capacity is associated with fast cycles of binding and dissociation, and thus c-Myc moves quickly across the nucleus (Fig. 2D, left).

In contrast to c-Myc, unbleached GFP-FoxA1 hardly reached the distal bleached region after 5 min (Fig. 2B,C). Even more strikingly, FoxA1 recovered from the border of the unbleached region first and then spread slowly into the bleached region (Fig. 2B). Note how the three different regions reached a plateau in their fluorescence recovery at different times for FoxA, but at similar times for c-Myc (Fig. 2C). The slow mode of nuclear movement for FoxA in the half-area FRAP assay is similar to what was seen previously for linker histone H1o (Phair et al. 2004) and differs from that of diverse other factors tested (Phair et al. 2004), including c-Myc, as shown here. Taken together, our results indicate that FoxAl's high nonspecific nucleosome- binding capacity is associated with slow cycles of binding and dissociation within the nucleus, and thus slow nuclear movement (Fig. 2D, right).

\section{DNA sequence-dependent and sequence-independent binding of FoxA1 to nucleosomes}

We next investigated in detail the target sequencedependent and sequence-independent binding of FoxA1 to nucleosomes. FoxA target sequences occur at approximately either half or one-and-a-half helical turns from the dyad axis on the N1 nucleosome at the alb1 enhancer in liver cells (McPherson et al. 1993; Chaya et al. 2001). By molecular modeling of the FoxA DBD on the NCP147 nucleosome structure at these two locations, we substituted a FoxA-binding sequence (FoxA-bds) into five positions of the NCP147 sequence (Fig. 3A,D; Supplemental Table 1; Luger et al. 1997). We note that the FoxA sequence was only the central 7 base pairs (bp) of the optimal full-length 15-bp sequence (Cirillo and Zaret 2007), in order to preserve the structure of the NCP147 on the nucleosome. Despite this, in EMSA, the nucleosomes with the FoxA-bds 2 bp downstream from the dyad axis $(D+2)$ showed enhanced binding to FoxA1 (Fig. $3 \mathrm{~B}, \mathrm{C}, P<0.01$ ), with an apparent $\mathrm{Kd}$ of $1.1 \mathrm{nM}$ for $\mathrm{D}+2$ compared with $1.8 \mathrm{nM}$ for the original NCP147 (Supplemental Fig. S3A,B). The D + 2 FoxA-bds is recognized by
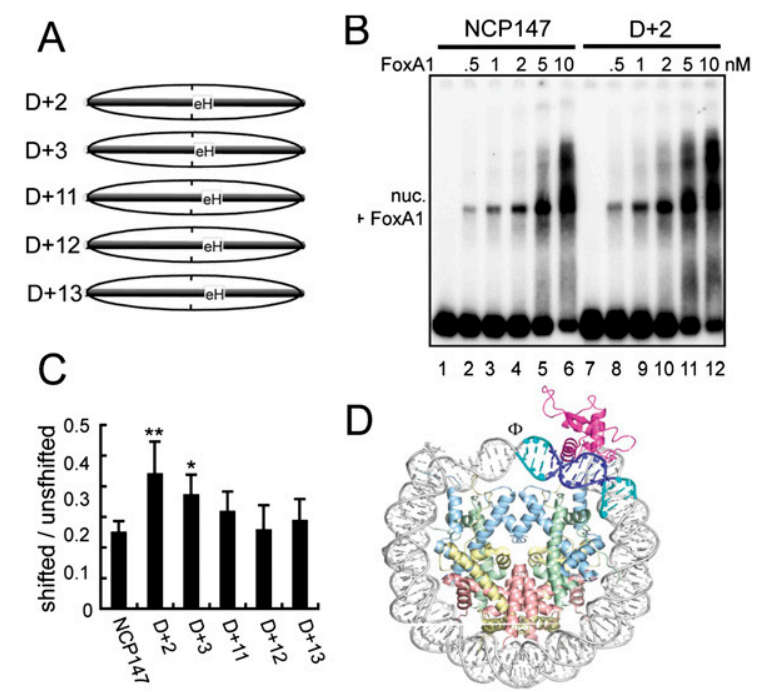

Figure 3. Specific and nonspecific binding of FoxA to mononucleosomes. (A) Schematic of NCP147 derivatives with an integrated eH FoxA-bds at indicated base pair positions with respect to the dyad axis $(D)$. (B) EMSA of NCP147 and its derivative D + 2 mononucleosomes with FoxA1. The FoxAl-probe complex position is shown. (C) Quantification of the EMSA in $B$. The ratio of shifted/ unshifted mononucleosomes at $2 \mathrm{nM}$ FoxAl is shown. Data are shown with the standard deviation $(\mathrm{SD}) ; n=3$; (*) $P<0.05$; (**) $P<$ 0.01 , Student's $t$-test. $(D)$ Modeling of the FoxA-DBD and the D +2 mononucleosome complex, by the program LSQMAN (see the Supplemental Material). The FoxA recognition site (TGTTTGC) is shown in dark blue; light blue shows the 13-bp fragment from the published FoxA-DNA structure (Clark et al. 1993). The hypothetical position of FoxA (based on superposition of the DNA part of the FoxA-DNA structure with the nucleosomal DNA) is shown in magenta. No clashes between the nucleosome and FoxA were observed in this or any other of the tested configurations, except with the very end of nucleosomal DNA. The nucleosomal dyad axis is indicated $(\Phi)$. 
FoxA1, as seen by DNase I footprinting (Supplemental Fig. S3C,D). These results indicate that the FoxA-bds enhances FoxA1 binding to NCP147 mononucleosomes nearly twofold and that, as observed for other factors (Li and Wrange 1995), the specificity is dependent on the translational and rotational position of the target sequence. It seems likely that a more native FoxA sequence would exhibit a greater affinity for the factor.

But more relevant to this study, the absence of FoxA1 footprints in bound complexes with the NCP147 nucleosomes (Supplemental Fig. S3C) shows that although the factor does not bind fortuitous DNA sites in that context, it still binds nonspecifically to the particles with relatively high affinity (Fig. 3C; Supplemental Fig. S3B).

\section{Sequence-nonspecific binding to nucleosomes} is a major determinant of FoxA1 movement in the nucleus

Previous studies in the FRAP field have argued that the relatively small number of functional target sites for a TF, relative to total genomic DNA, could not account for nuclear mobility dynamics of the factor (Karpova et al. 2004; Hinow et al. 2006). Yet a direct comparison of the specific and nonspecific binding of a factor to DNA or nucleosomes, in relation to nuclear mobility, has not been assessed. To directly assess this issue, we availed ourselves of the FoxA3-DNA crystal structure and the extreme conservation of the DBD sequence among FoxA family members to selectively mutate FoxA1's specific and nonspecific target site-binding capacity. The approach was enabled by the clear role of the FoxA DBD wing motifs in providing nonspecific interactions with the polynucleotide backbone of DNA, versus the HTH motif providing base-specific contacts within the double helix (Fig. 4A; Clark et al. 1993; Cirillo and Zaret 2007).

We made a FoxA NH-mut that contains alanine substitutions at two of the three amino acids within the HTH
(N216, H220) that make base contacts with DNA and thus is predicted to disrupt sequence-specific binding, while retaining some degree of nonspecific binding (Fig. 4A). We also made two different sets of wing mutations, each intended to disrupt sequence nonspecific binding, while retaining some degree of specific binding. Our RRmut alters two residues to alanines within wing 2 (R262, R265) that make phosphate contacts with DNA, and our SW-mut alters two residues to alanines within wing 1 (S242, W244) that make phosphate contacts with DNA (Fig. 4A). The occurrence of the RR and SW residues within random-coil segments of the protein diminishes the possibility that their mutation to alanines affects the overall fold of the DBD. The fact that the residues are buried within the DBD-DNA interaction surface diminishes the possibility that their mutation affects FoxA1 binding to other proteins.

We also made the compound NHRR-mut, which should disrupt both specific and nonspecific DNA binding, as well as the compound mut-All, which contains all three pairs of mutations described above. Recombinant proteins were made of each mutant (Supplemental Fig. S4A) for testing the above predictions in EMSA titration assays, and then the mutants were assessed in FRAP assays.

For EMSA, we used two pairs of DNA templates. The first pair consisted of the alb1 enhancer sequence N1-A, containing natural FoxA1-bds, and the N1-A eGeH-mut sequence, lacking FoxA1-bds (see Cirillo et al. 1998). The second pair of templates consisted of the $\mathrm{D}+2$ variant of the NCP147 sequence, containing an artificial FoxA1 site (Fig. 3A,D), and the NCP147 sequence, lacking such. Each pair of templates was used as free DNA and as assembled into mononucleosomes. The templates were used in EMSAs at $2 \mathrm{nM}$ concentration with increasing concentrations of recombinant FoxA1 proteins. Finally, the data were quantitated by PhosphorImager analysis and graphed to show the ratio of the first shifted band over unshifted
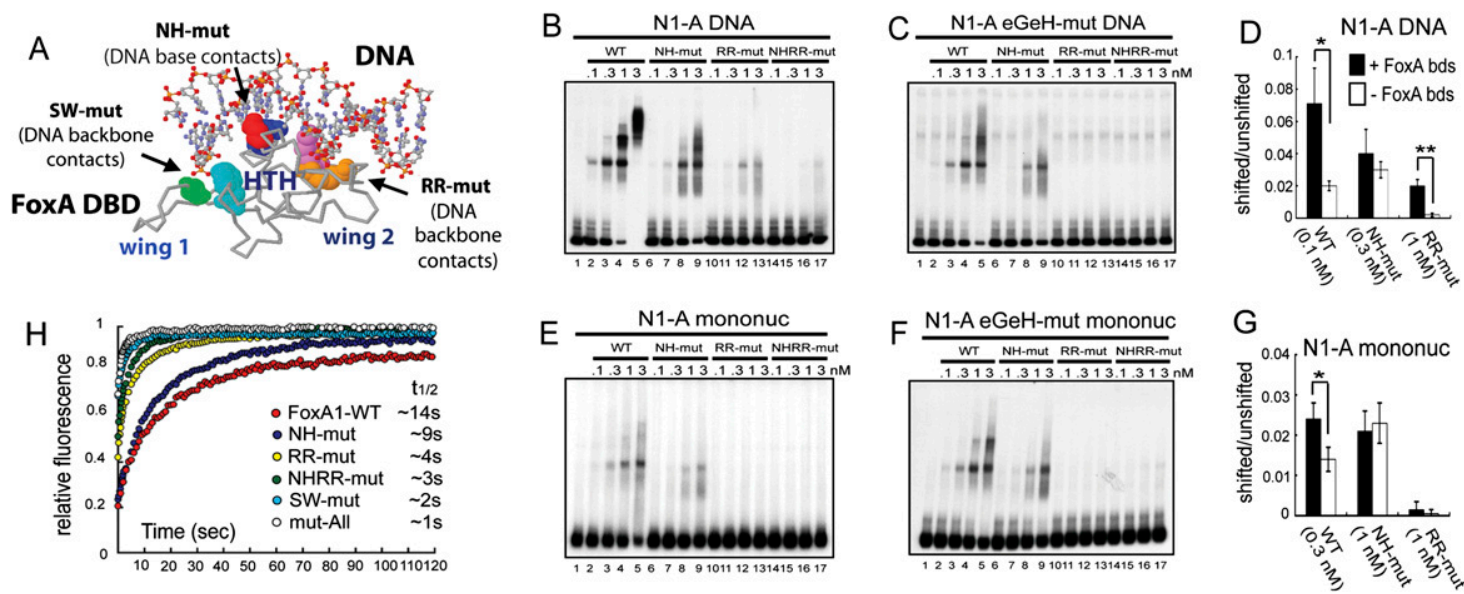

Figure 4. Sequence nonspecific interaction with DNA and nucleosomes is a major determinant of FoxAl movement in nucleus. $(A)$ Molecular modeling of the FoxA-DNA complex (Clark et al. 1993) using RASMOL. DNA in ball and stick, FoxA in gray wireframe, and amino acids mutated in spacefill. Amino acids are N216 (red), H220 (blue), R262 (orange), R265 (pink), S242 (green), and W244 (cyan). (B,C,E,F) EMSA with increasing concentrations of purified recombinant proteins of FoxA1 and the free N1-A DNA $(B, C)$ and mononucleosome $(E, F)$ probes indicated. Data from $B, C$, and $E, F$, where there is $>90 \%$ free probe and quantifiable shifting, are plotted in $D$ and $G$, respectively. N1-A DNAs or mononucleosomes either contained a FoxA-bds (solid bars), or lacked a FoxA-bds site (N1-A/eGeH mut, open bars). The data show that NH-mut is diminished in sequence-specific binding but retain nonspecific binding, and that RR-mut is diminished in overall nonspecific binding but retain some specific binding. $(H$, left $)$ Fluorescence recovery kinetics of GFP fusion FoxAl proteins indicated. The data show that loss of nonspecific binding (RR-mut) elicits a more dramatic increase in nuclear mobility than loss of specific binding (NH-mut). 
(free) template at FoxA1 concentrations where $>90 \%$ of template was free and where the shifted band was quantifiable (Fig. 4D,G; Supplemental Fig. 4D,G).

Wild-type FoxA1 showed the strongest binding to all of the DNAs and mononucleosomes, with an expected difference in affinity between templates with or without FoxA-bds (Fig. 4B-G; Supplemental Fig. S4B-G, lanes 1-5; $P \leq 0.05)$. The NH-mut, predicted to lose specific target binding, exhibited a several-fold decrease in binding to all of the DNA and mononucleosomes (Figs. 4B-G; Supplemental Fig. S4B-G, lanes 6-9|. But unlike wild-type FoxA1, the NH-mut did not exhibit a marked difference (by $t$-test) in affinity between templates containing versus lacking FoxA-bds. Furthermore, NH-mut retained considerable nucleosome binding.

In contrast, the RR-mut of wing 2 showed a greater decrease in binding to all DNA templates compared with the NH-mut (Fig. 4B,D; Supplemental Fig. S4B,C, lanes 10-13). Yet the RR-mut retained statistically significant, sequence-specific binding on the alb1 N1-A free DNA template, compared with the N1-A eGeH-mut (Fig. 4B-D, lanes $10-13 ; P \leq 0.05)$. More significantly, the RR-mut lost almost all binding to the nucleosomal templates (Fig. 4E-G; Supplemental Fig. S4E-G), demonstrating that nonspecific DNA binding is crucial for significant nucleosome binding.

The combined NHRR-mut showed an additional reduction of DNA and mononucleosome binding ability, completely losing consensus sequence recognition (Figs. 4B-G; Supplemental S4B-G, lanes 14-17). With the SWmut of wing 1, DNA binding was almost undetectable binding in the EMSA assay, consistent with a crucial role for wing 1 as described previously (Cirillo and Zaret 2007; data not shown). Finally, mutation of all six amino acids (mut-All) eliminated DNA and mononucleosome binding of FoxA1 (data not shown).

We next performed FRAP assays with the FoxA1 mutants fused to the same GFP as wild-type FoxA1, thus controlling for fluorescence quenching among the different constructs. All factors were nuclear and expressed at comparable levels (Supplemental Fig. 4H). The NH-mut, which lost sequence-specific binding but retained partial nonspecific binding to DNA and nucleosomes, exhibited an increase in nuclear mobility (Fig. $4 \mathrm{H}$, left panel). However, the NH-mut was distinctly the least affected of the mutants. The order of increasing nuclear mobility among the mutants was NH-mut, RR-mut, NHRR-mut, SW-mut, and mut-All. This corresponds precisely to the stepwise decrease in nonspecific DNA and nucleosome binding seen by the mutant series in vitro. Notably, the RR-mut, which retained free DNA binding but lost virtually all nucleosome binding in vitro, exhibited a more marked increase in nuclear mobility than the NH-mut. The mobility of mut-All was as fast as that of GFP fused to a nuclear localization signal (data not shown), which diffuses freely in the nucleoplasm (Phair et al. 2004). Based on the dramatic effects of the double amino acid changes on nonspecific nucleosome binding by FoxA1, we conclude that such binding is a major contributor to the factor's nuclear mobility.

\section{Nuclear mobility and pioneer factor function}

We speculate that the markedly higher affinity of FoxA for chromatin, compared with the behavior of c-Myc, reflects the enhanced ability of FoxA, in the half-area
FRAP assay, to scan both free DNA and nucleosomal target sequences (Fig. 3D, right). This has features similar to the role for nonspecific DNA interactions proposed to allow lac repressor and p53 to scan "linearly" along free DNA (Kalodimos et al. 2004; McKinney et al. 2004). Due to lower nucleosome affinity and less stable binding, cMyc can exist free in the nucleoplasm for longer periods than FoxA and hence move more quickly across the nucleus (Fig. 3D, left). By performing site-directed mutagenesis of the contact surface between FoxA1 and DNA, we discovered that the movement of FoxAl in the nucleus is restricted particularly by sequence-independent interactions with chromatin.

In cancer cell lines, FoxA1 preferentially associates with specific target sequences containing histone $\mathrm{H} 3$ that is dimethylated on Lys 4 (Lupien et al. 2008). Due to the statistical threshold to reduce the noise in their chromatin immunoprecipitation (ChIP)-chip analysis, Lupien et al. (2008) did not investigate nonspecific chromatin-binding events. However, we found that FoxA binds with high affinity to nucleosomes made with recombinant histones lacking eukaryotic modifications (Figs. 3, 4; Sekiya and Zaret 2007) and that the slow movement of FoxA in nuclei is limited by the factor's affinity toward nonspecific nucleosomal DNA. We speculate that the slow movement allows FoxA to locate high-affinity target sites in highly compacted chromatin in vivo, such as occurs at silent genes in development. For example, a GATA-4 site is occupied adjacent to a FoxA site at the silent alb1 gene in undifferentiated endoderm (Bossard and Zaret 1998). Yet GATA4 binding to nucleosomes is greatly facilitated by FoxA binding (Cirillo and Zaret 1999), and, as found here (Fig. 1A), GATA-4 is much more mobile in the nucleus than FoxA. We suggest that slow nuclear mobility reflects FoxA's function as a pioneer factor: probing "chromatinized" sites in nuclear DNA that many other TFs cannot access, followed by stable binding that allows other factors to engage the chromatin.

\section{Materials and methods}

Cells transfected with GFP fusion vectors were cultured on coverslips, then the coverslips were mounted on hanging drop slide glasses. Photobleaching was performed with a TCS SP2 confocal laser scanning microscope (Leica), using a 488-nm laser and a $63 \times$ plan Apo lens (1.4 NA). Laser power was attenuated to $1 \%$ of the bleach intensity. Five single-prebleach images were acquired, followed by four iterative bleach pulses of $241 \mathrm{msec}$ each. Single-section images were then collected at 0.241- to 1-sec intervals. Recovery of signal in the bleached region and loss of signal in the unbleached were taken as average intensities of at least $50 \%$ of the bleached or unbleached area. Recovery and loss curves were from background-subtracted images. Further details and other methods are in the Supplemental Material.

\section{Acknowledgments}

We thank Hua-Ying Fan and John Burch for comments on the manuscript, and Eileen Pytko and Sarah Costello-Berman for help in its preparation. T.S. was supported by a Post-doctoral Fellowship from the Japan Society for the Promotion of Science and the Uehara Memorial Foundation. The research was supported by grants from the NIH (R01/R37GM36477), the Mathers Charitable Foundation, and FCCC CCSG to K.S.Z.; by NIH GM061909 to K.L.; and by NIH GM077452 to A.V.T. 


\section{References}

Becker, M., Baumann, C., John, S., Walker, D.A., Vigneron, M., McNally, J.G., and Hager, G.L. 2002. Dynamic behavior of transcription factors on a natural promoter in living cells. EMBO Rep. 3: 1188-1194.

Bossard, P. and Zaret, K.S. 1998. GATA transcription factors as potentiators of gut endoderm differentiation. Development 125: 4909-4917.

Bossard, P. and Zaret, K.S. 2000. Repressive and restrictive mesodermal interactions with gut endoderm: Possible relation to Meckel's Diverticulum. Development 127: 4915-4923.

Buck, M.J. and Lieb, J.D. 2006. A chromatin-mediated mechanism for specification of conditional transcription factor targets. Nat. Genet. 38: 1446-1451.

Chaya, D., Hayamizu, T., Bustin, M., and Zaret, K.S. 2001. Transcription factor FoxA (HNF3) on a nucleosome at an enhancer complex in liver chromatin. J. Biol. Chem. 276: 44385-44389.

Cirillo, L.A. and Zaret, K.S. 1999. An early developmental transcription factor complex that is more stable on nucleosome core particles than on free DNA. Mol. Cell 4: 961-969.

Cirillo, L.A. and Zaret, K.S. 2007. Specific Interactions of the Wing Domains of FOXAl transcription factor with DNA. J. Mol. Biol. 366: 720-724.

Cirillo, L.A., McPherson, C.E., Bossard, P., Stevens, K., Cherian, S., Shim, E.-Y., Clark, E.A., Burley, S.K., and Zaret, K.S. 1998. Binding of the winged-helix transcription factor HNF3 to a linker histone site on the nucleosome. EMBO J. 17: 244-254.

Cirillo, L., Lin, F.R., Cuesta, I., Jarnik, M., Friedman, D., and Zaret, K. 2002. Opening of compacted chromatin by early developmental transcription factors HNF3 (FOXA) and GATA-4. Mol. Cell 9: 279-289.

Clark, K.L., Halay, E.D., Lai, E., and Burley, S.K. 1993. Co-crystal structure of the HNF-3/fork head DNA-recognition motif resembles histone H5. Nature 364: 412-420.

Davidson, E.H. and Erwin, D.H. 2006. Gene regulatory networks and the evolution of animal body plans. Science 311: 796-800.

Grange, T., Roux, J., Rigaud, G., and Pictet, R. 1991. Cell-type specific activity of two glucocorticoid responsive units of rat tyrosine aminotransferase gene is associated with multiple binding sites for C/EBP and a novel liver-specific nuclear factor. Nucleic Acids Res. 19: 131-139.

Gualdi, R., Bossard, P., Zheng, M., Hamada, Y., Coleman, J.R., and Zaret, K.S. 1996. Hepatic specification of the gut endoderm in vitro: Cell signaling and transcriptional control. Genes \& Dev. 10: 1670-1682.

Guccione, E., Martinato, F., Finocchiaro, G., Luzi, L., Tizzoni, L., Dall' Olio, V., Zardo, G., Nervi, C., Bernard, L., and Amati, B. 2006. Mycbinding-site recognition in the human genome is determined by chromatin context. Nat. Cell Biol. 8: 764-770.

Hinow, P., Rogers, C.E., Barbieri, C.E., Pietenpol, J.A., Kenworthy, A.K., and DiBenedetto, E. 2006. The DNA binding activity of p53 displays reaction-diffusion kinetics. Biophys. J. 91: 330-342.

Kalodimos, C.G., Biris, N., Bonvin, A.M., Levandoski, M.M., Guennuegues, M., Boelens, R., and Kaptein, R. 2004. Structure and flexibility adaptation in nonspecific and specific protein-DNA complexes. Science 305: 386-389.

Karpova, T.S., Chen, T.Y., Sprague, B.L., and McNally, J.G. 2004 Dynamic interactions of a transcription factor with DNA are accelerated by a chromatin remodeller. EMBO Rep. 5: 1064-1070.

Karpova, T.S., Kim, M.J., Spriet, C., Nalley, K., Stasevich, T.J., Kherrouche, Z., Heliot, L., and McNally, J.G. 2008. Concurrent fast and slow cycling of a transcriptional activator at an endogenous promoter. Science 319: 466-469.

Katoh, M. 2004. Human FOX gene family. Int. I. Oncol. 25: 1495-1500.

Kimura, H. and Cook, P.R. 2001. Kinetics of core histones in living human cells: Little exchange of $\mathrm{H} 3$ and $\mathrm{H} 4$ and some rapid exchange of H2B. J. Cell Biol. 153: 1341-1353.

Lee, C.S., Friedman, J.R., Fulmer, J.T., and Kaestner, K.H. 2005. The initiation of liver development is dependent on Foxa transcription factors. Nature 435: 944-947.

Lever, M.A., Th'ng, J.P., Sun, X., and Hendzel, M.J. 2000. Rapid exchange of histone H1.1 on chromatin in living human cells. Nature 408: 873876.

Li, Q. and Wrange, Ö. 1995. Accessibility of a glucocorticoid response element in a nucleosome depends on its rotational positioning. Mol. Cell. Biol. 15: 4375-4384.
Luger, K., Mader, A.W., Richmond, R.K., Sargent, D.F., and Richmond, T.J. 1997. Crystal structure of the nucleosome core particle at $2.8 \AA$ resolution. Nature 389: 251-260.

Lupien, M., Eeckhoute, J., Meyer, C.A., Wang, Q., Zhang, Y., Li, W., Carroll, J.S., Liu, X.S., and Brown, M. 2008. FoxA1 translates epigenetic signatures into enhancer-driven lineage-specific transcription. Cell 132: 958-970.

McKinney, K., Mattia, M., Gottifredi, V., and Prives, C. 2004. p53 linear diffusion along DNA requires its C terminus. Mol. Cell 16: 413-424.

McNally, J.G., Muller, W.G., Walker, D., Wolford, R., and Hager, G.L. 2000. The glucocorticoid receptor: Rapid exchange with regulatory sites in living cells. Science 287: 1262-1265.

McPherson, C.E., Shim, E.-Y., Friedman, D.S., and Zaret, K.S. 1993. An active tissue-specific enhancer and bound transcription factors existing in a precisely positioned nucleosomal array. Cell 75: 387-398.

Misteli, T., Gunjan, A., Hock, R., Bustin, M., and Brown, D.T. 2000. Dynamic binding of histone $\mathrm{H} 1$ to chromatin in living cells. Nature 408: $877-881$.

Nagaich, A.K., Walker, D.A., Wolford, R., and Hager, G.L. 2004. Rapid periodic binding and displacement of the glucocorticoid receptor during chromatin remodeling. Mol. Cell 14: 163-174.

Phair, R.D., Scaffidi, P., Elbi, C., Vecerova, J., Dey, A., Ozato, K., Brown, D.T., Hager, G., Bustin, M., and Misteli, T. 2004. Global nature of dynamic protein-chromatin interactions in vivo: Three-dimensional genome scanning and dynamic interaction networks of chromatin proteins. Mol. Cell. Biol. 24: 6393-6402.

Ramakrishnan, V., Finch, J.T., Graziano, V., Lee, P.L., and Sweet, R.M. 1993. Crystal structure of globular domain of histone H5 and its implications for nucleosome binding. Nature 362: 219-224.

Rayasam, G.V., Elbi, C., Walker, D.A., Wolford, R., Fletcher, T.M., Edwards, D.P., and Hager, G.L. 2005. Ligand-specific dynamics of the progesterone receptor in living cells and during chromatin remodeling in vitro. Mol. Cell. Biol. 25: 2406-2418.

Sekiya, T. and Zaret, K.S. 2007. Repression by Groucho/TLE/Grg proteins: Genomic site recruitment generates compacted chromatin in vitro and impairs activator binding in vivo. Mol. Cell 28: 291-303.

Simpson, R.T., Thoma, F., and Brubaker, J.M. 1985. Chromatin reconstituted from tandemly repeated cloned DNA fragments and core histones: A model system for study of higher order structure. Cell 42: 799-808.

Zaret, K.S. 2002. Regulatory phases of early liver development: Paradigms of organogenesis. Nat. Rev. Genet. 3: 499-512.

Zaret, K.S., DiPersio, C.M., Jackson, D.A., Montigny, W.J., and Weinstat, D.L. 1988. Conditional enhancement of liver-specific gene transcription. Proc. Natl. Acad. Sci. 85: 9076-9080. 


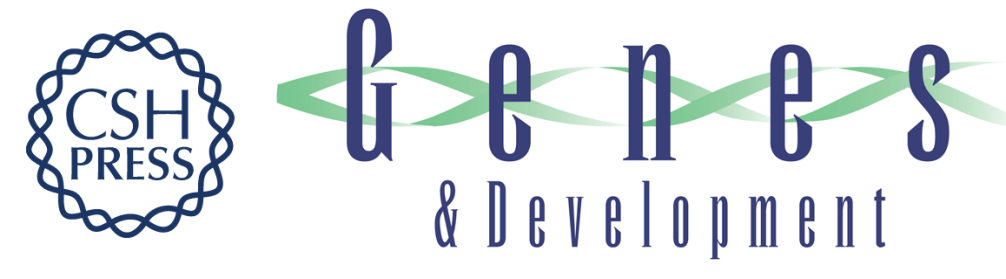

\section{Nucleosome-binding affinity as a primary determinant of the nuclear mobility of the pioneer transcription factor FoxA}

Takashi Sekiya, Uma M. Muthurajan, Karolin Luger, et al.

Genes Dev. 2009, 23:

Access the most recent version at doi:10.1101/gad.1775509

Supplemental
Material http://genesdev.cshlp.org/content/suppl/2009/04/02/23.7.804.DC1

References This article cites 37 articles, 14 of which can be accessed free at: http://genesdev.cshlp.org/content/23/7/804.full.html\#ref-list-1

License

Email Alerting

Receive free email alerts when new articles cite this article - sign up in the box at the top Service right corner of the article or click here.

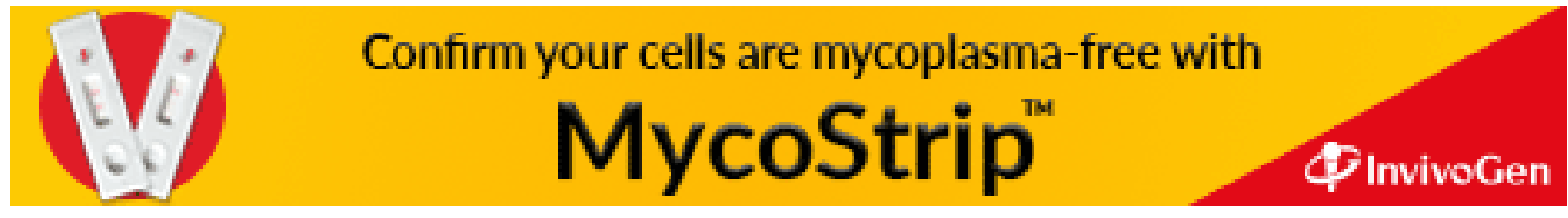

